\title{
Zero-norm states and stringy symmetries
}

\author{
Chuan-Tsung Chan ${ }^{1}$, Pei-Ming Ho ${ }^{2}$, Jen-Chi Lee ${ }^{3}$, Shunsuke \\ Teraguchi $^{1,2}$ and Yi Yang ${ }^{3}$ \\ ${ }^{1}$ Physics Division, National Center for Theoretical Sciences \\ ${ }^{2}$ Department of Physics, National Taiwan University, Taipei, Taiwan, R.O.C. \\ ${ }^{3}$ Department of Electrophysics, National Chiao-Tung University, Hsinchu, Taiwan, R.O.C. \\ E-mail: ctchan@phys.cts.nthu.edu.tw, pmho@ntu.edu.tw, jcclee@cc.nctu.edu.tw, \\ teraguch@phys.ntu.edu.tw, yiyang@mail.nctu.edu.tw
}

\begin{abstract}
We identify spacetime symmetry charges of 26D open bosonic string theory from an infinite number of zero-norm states (ZNS) with arbitrary high spin in the old covariant first quantized string spectrum. We give various evidences to support this identification. These include massive sigma-model calculation, Witten string field theory calculation, 2D string theory calculation and, most importantly, three methods of high-energy stringy scattering amplitude calculations. The last calculations explicitly prove Gross's conjectures in 1988 on high energy symmetry of string theory.
\end{abstract}

\section{Introduction and Overview}

One of the fundamental issues of string theory is the spacetime symmetry of the theory. It has long been believed that string theory consists of a huge hidden symmetry or Ward identities. This is strongly suggested by the ultraviolet finiteness of quantum string theory, which contains no free parameter and an infinite number of states. In a local quantum field theory, a symmetry principle was postulated, which can be used to determine the interaction of the theory. In string theory, on the contrary, it is the interaction, prescribed by the very tight quantum consistency conditions due to the extendedness of string, which determines the form of the symmetry.

Historically, the first key progress to understand symmetry of string theory is to study the high energy, fixed angle behavior of string scattering amplitude [1, 2, 3]. This is strongly motivated by the spontaneously broken symmetries in gauge field theories which are hidden at low energy, but become evident in the high-energy behavior of the theory. There are two main conjectures of Gross's [2] pioneer work on this subject. The first one is the existence of an infinite number of linear relations among the scattering amplitudes of different string states that are valid order by order in string perturbation theory at high energies. The second is that this symmetry is so powerful as to determine the scattering amplitudes of all the infinite number of string states in terms of the dilaton (tachyon for the case of open string) scattering amplitudes. However, the symmetry charges of his proposed stringy symmetries were not understood and the proportionality constants between scattering amplitudes of different string states were not calculated.

The second key to uncover the fundamental symmetry of string theory was zero-norm states (ZNS) in the old covariant first quantized (OCFQ) string spectrum. It was proposed that 
[4] spacetime symmetry charges of string theory orginate from an infinite number of ZNS with arbitrary high spin in the spectrum. In the context of $\sigma$-model approach of string theory, massive inter-particle symmetries were calculated by using two types of ZNS. Some implications of the corresponding stringy Ward identities on the scattering amplitudes were discussed in $[5,6]$. It was recently realized that $[7,8]$ these symmetries can be reproduced from gauge transformation of Witten string field theory (WSFT) [9] after imposing the no ghost conditions. It is important to note that these symmetries exist only for $\mathrm{D}=26$ thanks to type two ZNS, which is zero-norm only when $\mathrm{D}=26$. On the other hand, ZNS were also shown [10] to carry the spacetime $\omega_{\infty}$ symmetry [11] charges of 2D string theory [12]. This is in parallel with the work of Ref [13] where the ground ring structure of ghost number zero operators was identified in the BRST quantization. Other approaches of stringy symmetries include the studies of the high-energy, fixed momentum transfer regime [14], the Hagedorn transition at high temperature [15], vertex operator algebra for compatified spacetime or on a lattice [16], group theoretic approach of string [17] and by taking the tensionless limit of the worldsheet theory [18]. Despite all these developments, there seemed to be not much progress on this important subject.

Recently high-energy Ward identities derived from the decoupling of ZNS, which combines the previous two key ideas of probing stringy symmetry, were used to explicitly prove Gross's two conjectures [19, 20, 21, 22, 23]. An infinite number of linear relations among high energy scattering amplitudes of different string states were derived. Moreover, these linear relations can be used to fix the proportionality constants among high energy scattering amplitudes of different string states algebraically at each fixed mass level. Exactly the same results can also be obtained by two other calculations, the Virasoro constraint calculation and the saddle-point calculation. Thus there is only one independent component of high energy scattering amplitude at each fixed mass level. Based on this independent component of high energy scattering amplitude, one can then derive the general formula of high energy scattering amplitude for four arbitrary string states, and express them in terms of that of tachyons. This completes the general proof of Gross's two conjectures on high-energy symmetry of string theory stated above. Incidentally, it was important to discover $[19,20,21]$ that the result of saddle-point calculation in Refs $[1,2,3]$ was inconsistent with high energy stringy Ward identities of ZNS calculation in Refs $[19,20,21]$. A corrected saddle-point calculation was given in Ref [21], where the missing terms of the calculation in Refs $[1,2,3]$ were identified to recover the stringy Ward identities.

\section{Zero-norm state calculations}

In this section, we review the calculations of string symmetries from ZNS without taking the high-energy limit. In the OCFQ spectrum of open bosonic string theory, the solutions of physical states conditions include positive-norm propagating states and two types of ZNS. The latter are

Type I : $L_{-1}|x\rangle$, where $L_{1}|x\rangle=L_{2}|x\rangle=0, L_{0}|x\rangle=0$;

Type II : $\left(L_{-2}+\frac{3}{2} L_{-1}^{2}\right)|\widetilde{x}\rangle$, where $L_{1}|\widetilde{x}\rangle=L_{2}|\widetilde{x}\rangle=0,\left(L_{0}+1\right)|\widetilde{x}\rangle=0$.

While type I states have zero-norm at any space-time dimension, type II states have zero-norm only at $\mathrm{D}=26$. Some explicit solutions of ZNS can be found in [24]. In the $\sigma$-model approach of string theory, a spacetime symmetry transformation $\delta \Phi$ for a background field $\Phi$ can be generated by $[25]$

$$
T_{\Phi}+\delta T=T_{\Phi+\delta \Phi},
$$

where $T_{\Phi}$ is the worldsheet energy momentum tensor with background fields $\Phi$ and $T_{\Phi+\delta \Phi}$ is the new energy momentum tensor with new background fields $\Phi+\delta \Phi$. It was shown that [4] for each ZNS, one can construct a $\delta T$ such that Eq.(3) is satisfied to some order of weak field 
approximation in the $\beta$ function calculation. In constrast to the usual $\sigma$-model loop expansion (or $\alpha^{\prime}$ expansion), which is nonrenormalizable for the massive background field, it turns out that weak field approximation is the more convenient expansion to deal with massive background field. An inter-particle symmetry transformation for two high spin states at mass level $M^{2}=4$, for example, can be generated [4]

$$
\delta C_{(\mu \nu \lambda)}=\left(\frac{1}{2} \partial_{(\mu} \partial_{\nu} \theta_{\lambda)}-2 \eta_{(\mu \nu} \theta_{\lambda)}\right), \delta C_{[\mu \nu]}=9 \partial_{[\mu} \theta_{\nu]}
$$

where $\partial_{\nu} \theta^{\nu}=0,\left(\partial^{2}-4\right) \theta^{\nu}=0$ are the on-shell conditions of the mixed type I and type II $D_{2}$ vector ZNS

$$
\left|D_{2}\right\rangle=\left[\left(\frac{1}{2} k_{\mu} k_{\nu} \theta_{\lambda}+2 \eta_{\mu \nu} \theta_{\lambda}\right) \alpha_{-1}^{\mu} \alpha_{-1}^{\nu} \alpha_{-1}^{\lambda}+9 k_{\mu} \theta_{\nu} \alpha_{-2}^{[\mu} \alpha_{-1}^{\nu]}-6 \theta_{\mu} \alpha_{-3}^{\mu}\right]|0, k\rangle, \quad k \cdot \theta=0,
$$

and $C_{(\mu \nu \lambda)}$ and $C_{[\mu \nu]}$ are the background fields of the symmetric spin-three and antisymmetric spin-two states respectively at the mass level $M^{2}=4$. It is important to note that the decoupling of $D_{2}$ vector zero-norm state implies simultaneous change of both $C_{(\mu \nu \lambda)}$ and $C_{[\mu \nu]}$, thus they form a gauge multiplet. In WSFT, one can rederive [7, 8] Eq.(4) from the linearized off-shell gauge transformation

$$
\delta \Phi=Q_{\mathrm{B}} \Lambda
$$

after imposing the no ghost conditions.

Another evidence to support ZNS as the origin of symmetry charge was demonstrated for the $2 \mathrm{D}$ string theory. The spacetime symmetry of $2 \mathrm{D}$ string was known to be the $w_{\infty}$ algebra [11]

$$
\int \frac{d z}{2 \pi i} \psi_{J_{1} M_{1}}^{+}(z) \psi_{J_{2} M_{2}}^{+}(0)=\left(J_{2} M_{1}-J_{1} M_{2}\right) \psi_{\left(J_{1}+J_{2}-1\right)\left(M_{1}+M_{2}\right)}^{+}(0)
$$

generated by the discrete Polyakov states $\psi_{J M}^{+}$. An equivalent algebraic structure was the ground ring [13]

$$
Q_{J_{1}, M_{1}} \cdot Q_{J_{2}, M_{2}}=Q_{J_{1}+J_{2}, M_{1}+M_{2}}
$$

proposed by Witten. Alternatively, one can explicitly construct a set of discrete ZNS $G_{J M}^{+}$and show that they form a $w_{\infty}$ algebra [10]

$$
\int \frac{d z}{2 \pi i} G_{J_{1} M_{1}}^{+}(z) G_{J_{2} M_{2}}^{+}(0)=\left(J_{2} M_{1}-J_{1} M_{2}\right) G_{\left(J_{1}+J_{2}-1\right)\left(M_{1}+M_{2}\right)}^{+}(0) .
$$

This seems to strongly suggest that ZNS are closely related to the spacetime symmetry of string theory.

\section{High energy ZNS calculations}

Recently a further evidence to support ZNS as the spacetime symmetry charge of string theory was obtained by taking the high-energy, fixed angle limit of stringy Ward identities derived from the decoupling of ZNS on the scattering amplitudes. The two conjectures of Gross stated above were then explicitly proved. At mass level $M^{2}=4$, for example, the stringy Ward identities for four-point functions derived from the decoupling of four zero-norm states were calculated to be [5]

$$
\begin{gathered}
k_{\mu} \theta_{\nu \lambda} \mathcal{T}_{\chi}^{(\mu \nu \lambda)}+2 \theta_{\mu \nu} \mathcal{T}_{\chi}^{(\mu \nu)}=0 \\
\left(\frac{5}{2} k_{\mu} k_{\nu} \theta_{\lambda}^{\prime}+\eta_{\mu \nu} \theta_{\lambda}^{\prime}\right) \mathcal{T}_{\chi}^{(\mu \nu \lambda)}+9 k_{\mu} \theta_{\nu}^{\prime} \mathcal{T}_{\chi}^{(\mu \nu)}+6 \theta_{\mu}^{\prime} \mathcal{T}_{\chi}^{\mu}=0
\end{gathered}
$$




$$
\begin{gathered}
\left(\frac{1}{2} k_{\mu} k_{\nu} \theta_{\lambda}+2 \eta_{\mu \nu} \theta_{\lambda}\right) \mathcal{T}_{\chi}^{(\mu \nu \lambda)}+9 k_{\mu} \theta_{\nu} \mathcal{T}_{\chi}^{[\mu \nu]}-6 \theta_{\mu} \mathcal{T}_{\chi}^{\mu}=0 \\
\left(\frac{17}{4} k_{\mu} k_{\nu} k_{\lambda}+\frac{9}{2} \eta_{\mu \nu} k_{\lambda}\right) \mathcal{T}_{\chi}^{(\mu \nu \lambda)}+\left(21 k_{\mu} k_{\nu}+9 \eta_{\mu \nu}\right) \mathcal{T}_{\chi}^{(\mu \nu)}+25 k_{\mu} \mathcal{T}_{\chi}^{\mu}=0
\end{gathered}
$$

where $\theta_{\mu \nu}$ is symmetric, transverse and traceless, and $\theta_{\lambda}^{\prime}$ and $\theta_{\lambda}$ are transverse vectors. These are polarizations of zero-norm states. $\mathcal{T}_{\chi}^{\prime} s$ in Eqs.(10)-(13) are $\chi$-th order string-loop amplitudes. In each equation, we have chosen, say, the second vertex $V_{2}\left(k_{2}\right)$ in the correlation function to be constructed from zero-norm states at the mass level $M^{2}=4$ and $k_{\mu} \equiv k_{2 \mu}$. The tensor index of the other three string vertex were suppressed in Eq.(10)-(13). By using Eqs.(10)-(13), the leading order Ward identities of Eqs.(10)-(13) in the $\frac{1}{E^{2}}$ expansions were calculated to be (we drop loop order $\chi$ here to simplify the notation) $[19,20]$

$$
\begin{gathered}
\mathcal{T}_{L L T}+\mathcal{T}_{(L T)}=0 \\
10 \mathcal{T}_{L L T}+\mathcal{T}_{T T T}+18 \mathcal{T}_{(L T)}=0 \\
\mathcal{T}_{L L T}+\mathcal{T}_{T T T}+9 \mathcal{T}_{[L T]}=0 .
\end{gathered}
$$

In the above equations, we have defined the normalized polarization vectors of the second string vertex to be $e_{P}=\frac{1}{m_{2}}\left(E_{2}, \mathrm{k}_{2}, 0\right)=\frac{k_{2}}{m_{2}}, e_{L}=\frac{1}{m_{2}}\left(\mathrm{k}_{2}, E_{2}, 0\right)$ and $e_{T}=(0,0,1)$ in the CM frame contained in the plane of scattering. It turns out that $e_{P}$ approaches $e_{L}$ in the high-energy limit. A simple calculation shows that, in contrast to Eq.(4) which is valid to all energies, the linear relations among the high-energy scattering amplitudes are [19, 20]

$$
\mathcal{T}_{T T T}: \mathcal{T}_{L L T}: \mathcal{T}_{(L T)}: \mathcal{T}_{[L T]}=8: 1:-1:-1
$$

Note that the underlying high-energy limit of zero-norm states in the equations above are no longer of zero norm. That is why we will be able to obtain nontrivial relations among physically inequivalent particles. For the case of string-tree level $\chi=1$ with one tensor $V_{2}$ and three tachyons $V_{1,3,4}$, all four scattering amplitudes in Eq.(17) were calculated to be $\mathcal{T}_{T T T}=-8 E^{9} \sin ^{3} \phi_{C M} \mathcal{T}(3)=8 \mathcal{T}_{L L T}=-8 \mathcal{T}_{(L T)}=-8 \mathcal{T}_{[L T]}$, where

$$
\begin{aligned}
\mathcal{T}(n)= & \sqrt{\pi}(-1)^{n-1} 2^{-n} E^{-1-2 n}\left(\sin \frac{\phi_{C M}}{2}\right)^{-3}\left(\cos \frac{\phi_{C M}}{2}\right)^{5-2 n} \\
& \times \exp \left(-\frac{s \ln s+t \ln t-(s+t) \ln (s+t)}{2}\right)
\end{aligned}
$$

In Eq.(18), $\phi_{C M}$ is the center of momentum scattering angle, $s, t$ and $u$ are the Mandelstam variables and $M^{2}=2(n-1)$.

For the scattering amplitude of general mass level, one first notes that the only states that will survive in the high energy limit at level $M^{2}=2(n-1)$ are of the form

$$
|n, 2 m, q\rangle \equiv\left(\alpha_{-1}^{T}\right)^{n-2 m-2 q}\left(\alpha_{-1}^{L}\right)^{2 m}\left(\alpha_{-2}^{L}\right)^{q}|0, k\rangle .
$$

The next step is to use the decoupling of two types of high-energy ZNS

$$
\begin{gathered}
L_{-1}|n-1,2 m-1, q\rangle \simeq M|n, 2 m, q\rangle+(2 m-1)|n, 2 m-2, q+1\rangle, \\
L_{-2}|n-2,0, q\rangle \simeq \frac{1}{2}|n, 0, q\rangle+M|n, 0, q+1\rangle,
\end{gathered}
$$

to deduce that $[22,23]$

$$
\mathcal{T}^{(n, 2 m, q)}=\left(-\frac{1}{M}\right)^{2 m+q}\left(\frac{1}{2}\right)^{m+q}(2 m-1) ! ! \mathcal{T}^{(n, 0,0)}
$$


For the case of general four tensor scattering amplitude, one has, in the high energy limit,

$$
<V_{1} V_{2} V_{3} V_{4}>=\prod_{i=1}^{4}\left(-\frac{1}{M_{i}}\right)^{2 m_{i}+q_{i}}\left(\frac{1}{2}\right)^{m_{i}+q_{i}}\left(2 m_{i}-1\right) ! ! \mathcal{T}_{n_{1} n_{2} n_{3} n^{1} \cdot T_{4}^{2} . \cdot T^{3} \cdot .},
$$

which is calculated algebraically by the decoupling of high-energy ZNS and is thus valid to all string-loop order. $\mathcal{T}_{n_{1} n_{2} n_{3} n_{4} n^{1} . T^{2} . T^{4} . .}$ in Eq.(23), the generalization of $\mathcal{T}_{T T T}$ at mass level $M^{2}=4$, is the only independent high-energy scattering amplitudes at level $\left(n_{1}, n_{2}, n_{3}, n_{4}\right)$ and was calculated at tree level to be $[19,21]$

$$
\mathcal{T}_{n_{1} n_{2} n_{3} n_{4} \cdot n^{2} . T^{3} . T^{4} . .}^{n^{2}}=\left[-2 E^{3} \sin \phi_{C M}\right]^{\Sigma n_{i}} \mathcal{T}\left(\Sigma n_{i}\right)=2 \sqrt{\pi} e^{n-4}(s t u)^{\frac{n-3}{2}} e^{-\frac{1}{2}(s \ln s+t \ln t+u \ln u)},
$$

where $n_{i}$ is the number of $T^{i}$ of the $i-t h$ vertex operators, $n=\sum_{i=1}^{4} n_{i}$ and $T^{i}$ is the transverse direction of the $i-t h$ particle.

The decoupling of two types of high-energy ZNS in Eqs.(20) and (21) can be shown to be equivalent to the decoupling of two types of zero-norm states in Eqs.(1) and (2) in the highenergy limit. It is interesting to see that the second term $\frac{3}{2} L_{-1}^{2}|\widetilde{x}\rangle$ of type II zero-norm states in Eq.(2) can be dropped out in the high-energy limit without affecting the final result. This hints at a "dual" calculation, the high-energy limit of the Virasoro constraints $L_{1}|\psi\rangle=L_{2}|\psi\rangle=0$, to derive Eq.(22) [22, 23]. Finally, a saddle-point calculation [21, 22, 23] can be developed for the string-tree level $\chi=1$ scattering amplitudes with one tensor $V_{2}$ and three tachyons $V_{1,3,4}$ to explicitly justify Eq.(22).

For the case of $2 \mathrm{D}$ string theory, The discrete $2 \mathrm{D}$ ZNS $G_{J M}^{+}$constructed in [10] can be shown [22] to approach to the discrete Polyakov states $\psi_{J M}^{+}$in the high-energy limit. Thus the $w_{\infty}$ algebra constructed in Eq.(9) can be identified to that of Eq.(7) in the high-energy limit.

\section{References}

[1] D.J. Gross and P. Mende, Phys. Lett. B197,129 (1987); Nucl.Phys.B303, 407(1988).

[2] D.J. Gross, "High energy symmetry of string theory", Phys. Rev. Lett. 60,1229 (1988); Phil.Trans. R. Soc. Lond. A329,401(1989).

[3] D.J. Gross and J.L. Manes, "The high energy behavior of open string theory", Nucl.Phys.B326, 73(1989). See section 6 for details.

[4] J.C. Lee, Phys. Lett. B241, 336(1990); J.C. Lee, Phys. Rev. Lett. 64, 1636(1990); J.C. Lee and B. Ovrut, Nucl. Phys. B336, 222(1990).

[5] J.C. Lee, Prog. of Theor. Phys. Vol. 91, 353(1994).

[6] J.C. Lee, Phys. Lett. B337,69(1994).

[7] H.C. Kao and J.C. Lee, Phys. Rev. D67, 086003(2003).

[8] C.T. Chan, J.C. Lee and Y. Yang, "Anatomy of zero-norm states in string theory", Phys. Rev.D71, 086005 (2005), hep-th/0501020.

[9] E. Witten, Nucl. Phys. B268, 253 (1986).

[10] T. D. Chung and J. C. Lee, "Discrete gauge states and $w_{\infty}$ charges in $c=12 D$ gravity" Phys. Lett. B350, 22 (1995) [arXiv:hep-th/9412095]; "Superfield form of discrete gauge states in c $=12$-d supergravity" Z. Phys. C 75, 555 (1997) [arXiv:hep-th/9505107]. J.C. Lee, Euro.Phys. JC1,739(1998).

[11] J. Avan and A.Jevicki, Phys. Lett. B266, 35 (1991); B272, 17 (1991). I.R. Klebanov and A.M. Polyakov, Mod. Phys. Lett. A6, 3273 (1991).

[12] For a review see I.R. Klebanov and A. Pasquinucci, hep-th/9210105 and references therein.

[13] E. Witten, Nucl. Phys. B373, 187 (1992); E.Witten and B. Zwiebach, Nucl. Phys. B377, 55 (1992).

[14] D. Amati,M. Ciafaloni and G.Veneziano, Phys. Lett. B216, 41 (1989).

[15] J.J. Atick and E. Witten, Nucl. Phys. B310, 291 (1988); R. Hagedorn, Nuovo Cimento Supplement, 3,147 (1965).

[16] C.W. Moore, hep-th/9305139, hep-th/9310026.

[17] P.C. West, Mod. Phys. Lett. A10, 761(1995), N. Moeller and P. C. West, "Arbitrary four string scattering at high energy and fixed angle", Nucl. Phys. B729, 1(2005), hep-th/0507152.

[18] See, for instance, J. Isberg, U. Lindstrom, B. Sundborg and G. Theodoridis, Nucl. Phys. B 411, 122 (1994) [arXiv:hep-th/9307108]; B. Sundborg, Nucl. Phys. Proc. Suppl. 102, 113 (2001) [arXiv:hep-th/0103247]; 
E. Sezgin and P. Sundell, Nucl. Phys. B 644, 303 (2002) [Erratum-ibid. B 660, 403 (2003)] [arXiv:hepth/0205131]; C. S. Chu, P. M. Ho and F. L. Lin, JHEP 0209, 003 (2002) [arXiv:hep-th/0205218]; G. Bonelli, Nucl. Phys. B 669, 159 (2003) [arXiv:hep-th/0305155].

[19] C.T.Chan and J.C. Lee, "Stringy symmetries and their high-energy limits", Phys. Lett. B611,193 (2005), hep-th/0312226. J.C. Lee, hep-th/0303012.

[20] C.T. Chan and J.C. Lee, Nucl.Phys. B690 (2004)3, hep-th/0401133.

[21] C. T. Chan, P. M. Ho and J. C. Lee, "Ward identities and high-energy scattering amplitudes in string theory," Nucl. Phys. B 708, 99 (2005) [arXiv:hep-th/0410194].

[22] C.T. Chan, P.M. Ho, J.C. Lee, S. Teraguchi and Y. Yang, " Solving all 4-point correlation functions for bosonic open string theory in the high energy limit", Nucl.Phys.B725, 352(2005). hep-th/0504138.

[23] C.T. Chan, P.M. Ho, J.C. Lee, S. Teraguchi and Y. Yang, "High-energy zero-norm states and symmetries of string theory". hep-th/0505035.

[24] J.C. Lee, "Calculations of zero-norm states and reduction of stringy scattering amplitudes", Prog. Theo. Phys. 114, 259(2005), hep-th/0302123.

[25] M. Evans and B.A. Ovrut, Phys. Lett. B231,80 (1989). 